\title{
ÜBER DEN TRICHTERAPPARAT DER LARVEN VON MEGALOPHRYS MONTANA KUHL
}

VON

\author{
Dr. H. BOSCHMA.
}

(Mit 1 Figur im Tekst).

\begin{abstract}
Während eines Aufenthaltes in Tjibodas war ich in der Lage öfters die Larven von Megalophrys montana zu bekommen. Prof. Weber beschrieb diese eigentümlichen Larven zuerst (1888) von demselben Fundort, während sie später auch anderswo auf Java (VAN KAMPEN 1909) und auf der malayischen Halbinsel (ANNANDALE 1903) gefunden wurden ').

In Tjibodas und in der Umgebung fand ich diese Larven an ruhigeren Stellen schnellfliessender wasserreicher Bäche, in einem beinahe trockenen Tümpel, und in einem Sumpfe mit sehr langsam strömendem Wasser und dichter Vegetation. Die Höhe der Fundorte variierte von etwa 1400 bis 1600 Meter, sie befinden sich alle im Urwalde. In Dezember, Januar und April fand ich jedesmal Larven von $23 \mathrm{~mm}$. Totallänge bis Larven mit wohlentwickelten Vorderbeinen, jüngere Entwickelungsstadien habe ich leider nicht auffinden können. Auffallend war der Grössenunterschied zwischen den Larven aus dem Sumpfe und denen von anderen Fundorten. Während die grösseren Larven von den anderen Fundorten, bei denen die Bildung der Hinterbeine schon angefangen hatte, keine grössere Länge erreichten als $54 \mathrm{~mm}$., waren die Larven aus dem Sumpfe viel grösser und kräftiger, sie erreichten eine Totallänge von $62 \mathrm{~mm}$. WEBER gibt für die Totallänge dieser Larven $45 \mathrm{~mm}$. an, vAN KAMPEN fand Larven bis zu 54.5 mm., während die Länge der grössten Larve von der malayischen Halbinsel nach BOULENGER (1912) ungefähr $40 \mathrm{~mm}$. betrug. Nur in diesem Sumpfe fand ich diese kräftigen Larven, und hier kamen keine gewöhnlichen, kleineren Larven vor.

WEBER hat den trichterförmigen Mundapparat dieser Larven anatomisch untersucht und die Lebensweise der Larven studiert, während ANNANDALE später (1903) eine Untersuchung über denselben Gegenstand veröffentlichte und zu einigermassen abweichenden Resultaten kam. Während der Zeit die ich in Tjibodas verblieb, habe ich die Larven gezüchtet und versucht etwas näheres über die Lebensweise kennen zu lernen. Einige (etwa 15) Larven wurden aus einem schnellfliessenden Bache sofort in ein Glas von etwa 2 Liter Inhalt übergeführt. Von diesem plötzlichen Uebergang in ein ganz verschiedenes Milieu schienen sie nicht ungünstig beeinflusst $\mathrm{zu}$ werden, denn sie verhielten sich ganz normal. Nachdem sie einige Minuten hin- und hergeschwommen waren, blieben einige am Boden. ruhig atmend liegen, während andere mit ausgebreitetem Trichterapparat an der Oberfläche hingen. Als Nahrung bekamen sie Fadenalgen (Spirogyra u. a.) aus einem Teiche, also auch etwas anderes als sie zu fressen gewohnt waren, denn in den Bächen, wo ich die Larven fischte, kommen keine solche Algen vor. Trotzdem sie in sehr veränderte Umstände kamen, blieben sie sehr lebhaft und entwickel-
\end{abstract}

1) Die Larven, welche ANNANDALE (1906) im Himalaya-Gebiet fand, gehören nach Boulenger (1908) zu Megalophrys parva. 
ten sich weiter bis der Schwanz beinahe resorbiert worden war. Weiter konnte ich sie nicht züchten; denn die jungen Tiere starben nach ein Paar Tagen. Die ungewohnte Nahrung schien keinen hemmenden Einfluss auf die Entwickelung auszuüben.

Die Körperform der Larven ist eine Anpassung an das Leben in schnellfliessendem Wasser, sie haben einen sehr kräftigen Ruderschwanz (vergl. GaDow's Fig. 11, in WeBER's Fig. 1 und 2 sind sie mit zu kurzem Schwanze abgebildet). Obwohl die Wasseroberfläche sich etwa $10 \mathrm{~cm}$. unter dem oberen Rande des Behälters befand, konnten sie doch herausspringen, was später vermieden wurde indem ein Gazetuch über den Behälter gespannt wurde. Ein viertelstündiges Verbleiben ausserhalb des Wassers schadete jedoch den Larven nicht, in das Wasser zurückgebracht schwammen sie wieder lebhaft umher. Weil ich diese Larven sogar unter sehr veränderten Umständen so leicht züchten konnte, wundert es mich, dass ANnANDAle sie nicht länger als etwa zwei Tage am Leben behalten konnte (vergl. BOULENGER 1903), denn meine Larven lebten mehr als drei Wochen in diesem kleinen Raum.

Wie es schon WeBER und ANNANDale hervorheben, kann der trichterförmige Mundapparat der Megalophrys-Larven zwei Formen annehmen. Wenn die Larve an der Oberfläche des Wassers schwimmt, ist der Trichter an seiner Peripherie flach auf dem Wasser ausgebreitet, während der zentrale Teil, wo sich die Mundöffnung befindet, nach innen eingesenkt ist. Der Körper hängt dann, wenn der Mundapparat nicht in Berührung mit irgend einem festen Gegenstand ist, unter einem Winkel von etwa 45 Grad im Wasser. Wenn aber die ventrale Lippe des Trichters die Glaswand berührt, so nehmen die Tiere einen vertikaleren Stand ein ${ }^{1}$ ). Sie sind im Stande langsam zu schwimmen mit ausgebreitetem Trichter, dann wird der Winkel zwischen der Wasseroberfläche und der Körperachse ein kleinerer. Bewegen sie sich schneller, so tauchen sie unter und der Trichter faltet sich einigermassen zusammen, wie es auch der Fall ist, wenn die Tiere ruhig am Boden liegen. Dann sind aber die Lippen nicht geschlossen, es findet nur eine Krümmung der peripheren Teile nach innen statt. Niemals habe ich einen so weitgehenden Verschluss des Mundapparates konstatieren können wie es ANNANDALE beschreibt und im Medianschnitt abbildet, es blieb immer noch eine weite Oeffnung übrig, auch bei den (in Formol) fixierten Exemplaren.

Wenn nun das auf dem Boden liegende Tier nach oben schwimmt und mit dem Mundapparat die Oberfläche berührt, so sieht man, dass sich der Trichter sofort an dem Wasserspiegel ausbreitet. In den Lippen befinden sich einige Muskelbündel, welche nach WEBER durch ihre Kontraktion Verschluss der Lippenränder herbeiführen. ANNANDALE vertritt die entgegengesetzte Meinung: die Muskelkontraktion würde die Ausbreitung des basalen Teiles des Trichters zustandebringen. Diese Frage von der Bedeutung der Trichtermuskeln habe ich zu lösen versucht indem ich in einem kleinen Behälter mit einigen Larven ein wenig Strychnin löste, das bekanntlich Muskelkontraktion hervorruft. Einige Larven, die am Boden lagen, wurden zuerst unruhig, schwammen dann umher und versuchten zuletzt vergeblich ihren Trichterapparat an der Wasseroberfläche auszubreiten. Sie fuhren mit der Schnauze gegen die Oberfläche, aber der zusammengefaltete Mundapparat konnte sich nicht ausbreiten und sie sanken wieder zurück. Von einigen anderen Larven, welche beim Anfang des Versuchs mit ausgebreitetem Trichter an der Wasseroberfläche hingen, wurde die Oeffnung des Trichters allmählich kleiner, bis auch sie zu Boden sanken; als sie wieder nach oben schwammen, konnten sie den Trichter nicht mehr entfalten. Aus diesem Versuch geht somit hervor, dass der Trichter durch die Kontraktion der Muskeln zusammengezogen wird. Kommt dann das Tier mit seiner Schnauze an die Wasseroberfläche, so geben die Muskeln nach, und der Trichter breitet sich aus, vielleicht auch noch mit Hülfe der Oberflächenspannung des Wassers.

Hinsichtlich der Funktion des Trichters dieser Larve sagt WeBer: „am Rande des Baches erhebt sie sich bis zur Oberfläche des Wassers in der Art, dass der weitgeöffnete Rüssel genau an die Oberfläche des Wassers zu liegen kommt und somit treibendes mikroskopisches Material aufnehmen kann" (1888, S. 10). Weiter würden kleine Organismen und zarte Pflanzenteile zwischen den beiden Lippen mit Hülfe der auf denselben sich befindenden Erhebungen zerrieben und der Mundöffnung

1) Die Stellung des ruhig im Wasser hangenden Tieres wird oft ungenau angegeben, nach ANNANDALE (1903) hängen sie "more or less vertically" nach WERNER (1912) "fast senkrecht". WEBER (1888) hingegen gibt eine gute schematische Figur (Fig. 5). 
zugeschoben werden. Ich habe dies nicht bestätigen können, da bei meinen Larven die Lippen sich nie berührten, immer blieb ein breiter Spalt zwischen Ober- und Unterlippe. ANNANDALE beschreibt die Funktion des Trichters mit folgenden Worten: "The main function of the funnel, then, is to act as a float, and, very probably, to protect the mouth of the tadpole from the entry of noxious organisms when it is burrowing in the mud, as drought causes it to do. It is possibly also that it assists in the capture of the minute plants and animals on which the species feeds, though I can give no direct evidence for this function. If the tadpole is watched when it is hanging from the surface film, it will be remarked that the float is constantly expanding and contracting, owing to the respiratory movements of the mouth, and that, although the movements are too gentle to overcome the friction produced by the body and tail, which hang more or less vertically downwards in the water, and so to cause the animal to progress in one direction or another, they produce distinct currents on the surface, which bring towards the mouth any minute particle which may chance to be floating in its vicinity". (1903, S. 279, 280).

Wenn die Larve mit ausgebreitetem Trichter an der Oberfläche des Wassers hängt, liegt dieser Apparat fast regungslos, nur bewegt sich die Unterlippe ganz leise, der Atembewegungen des Tieres zufolge. Weil nun gewöhnlich sehr wenig mikroskopisches Material an der Oberfläche treibt, habe ich ein wenig Karminpulver sich in der Nähe der Larven auf dem Wasser ausbreiten lassen und konnte nun sehr schön sehen, welche Strömungen von den Atembewegungen der Larven an der Oberfläche des Wassers verursacht wurden (vergl. Tekstfigur). Das Karminpulver an der kaudalen Seite des

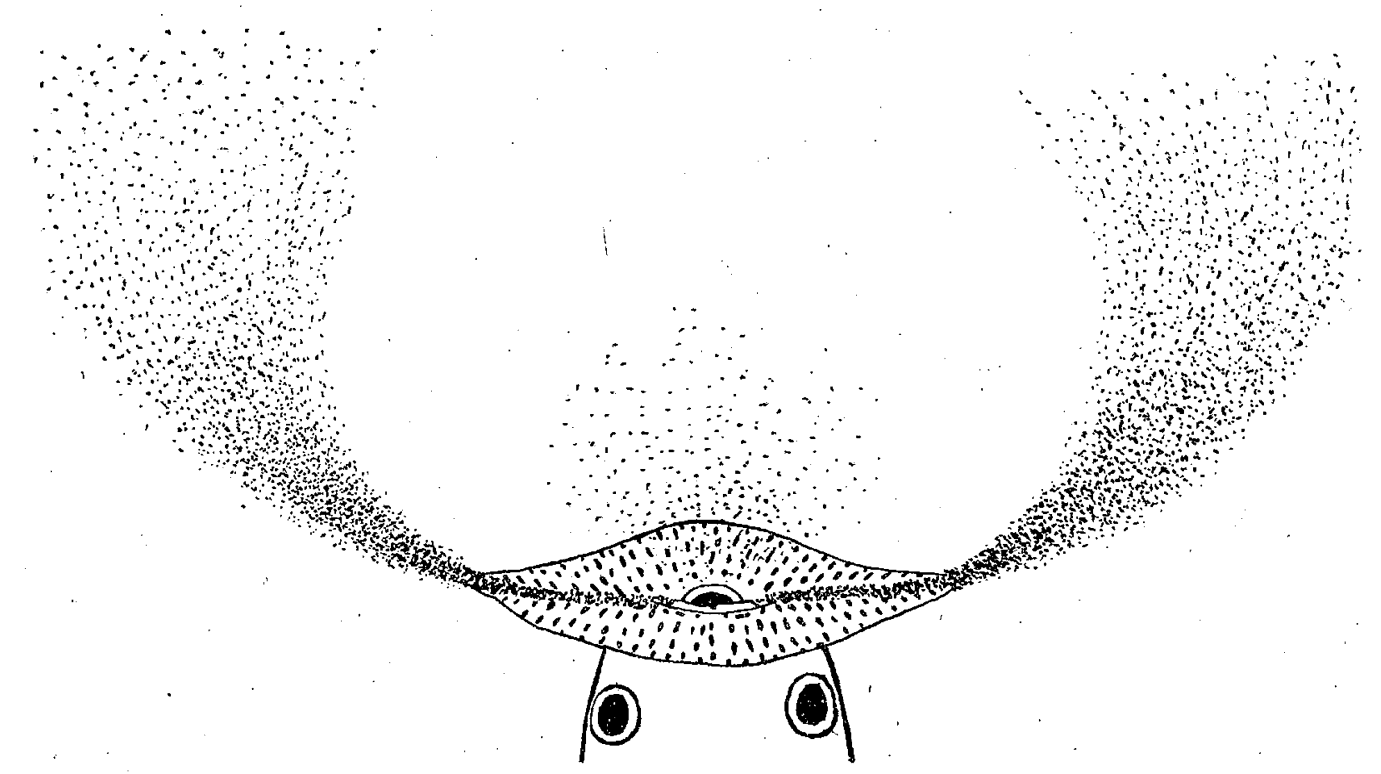

Vorderer Teil einer Larve von Megalophrys montana, ruhig atmend an der Wasseroberfläche hängend. Auf dem Wasser ist Karminpulver ausgebreitet, nur die sich bewegenden Karminteilchen sind gezeichnet. Vergl. weiter den Tekst. \pm 5 mal vergr.

Trichters blieb in Ruhe, aber vor dem Trichter waren zwei starke Strömungen zu beobachten, welche das Pulver genau nach den seitlichen Ecken des Trichters führten. An der Grenze zwischen Oberund Unterlippe befindet sich eine Strecke, wo die Erhebungen fehlen, wie schon WeBER nachwies, und genau dieser Grenze entlang wurde das Pulver zum Munde geführt. Ferner war noch eine schwächere Strömung vor dem Munde zu sehen, wo ganz langsam das Pulver über den Rand der Unterlippe herausbefördert wurde. Die Menge des aufgenommenen und des ausgestossenen Pulvers war sehr verschieden: allmählig sammelte sich ein kleiner Haufen Karminpulver vor dem Munde, denn nur eine ganz geringe Menge geriet in die Atemhöhle. Schliesslich schien sich zu viel Pulver vor dem Munde der Larven angesammelt $\mathrm{zu}$ haben, dann wurde es, oft $10 \mathrm{~cm}$. weit, über die Unterlippe nach vorne ausgespuckt. Gewöhnlich schwamm die Larve darauf eine kurze Strecke weiter, wo sich der Prozess wiederholte.

Im Freien kann auf diese Weise die Larve, wenn sie ruhig atmend an der Wasseroberfläche hängt, die mikroskopischen treibenden Teilchen, sei es Detritus oder lebende Pflanzen oder Tiere, 
aufnehmen. Wenn mit dem Wasserstrom etwas. Unessbares dem Munde zugeführt wird, so kann sie es mittels eines starken Wasserstroms aus dem Munde herausbefördern.

Die Erhebungen auf der Innenseite des Trichters haben nach den meisten Autoren die Bedeutung kleine Teilchen festzuhalten oder sogar zu zerreiben. Dieser Meinung kann ich nicht beipflichten, weil diese Erhebungen nicht verhornt sind, sondern nur Verdickungen der Lederhaut, welche von einer einzelligen Epithelschicht bedeckt sind, wie WEBER richtig beschrieb. Daher dürfen diese Organe nicht „Hornzähne” genannt werden, wie es öfters geschieht, denn die echten Hornzähne dieser Larven befinden sich in einer Reihe am Oberkiefer. Die Form der Erhebungen ist stumpfkonisch am Rande des Mundes bis langgestreckt am grössten Teile des Trichters. Nie fand ich sie so scharf zugepitzt wie. sie ANNANDale abbildet.

Im Darm der Larven fand ich sehr viele Gewebsstücke höherer Pflanzen, einige kleine Crustaceen, Beinstücke von Copepoden oder Milben, viele Diatomeen aus verschiedenen Gattungen, besonders die kleineren Formen in grosser Anzahl, ein paar Stücke von Fadenalgen, Infusorien und Dauerzustände von einzelligen Organismen. Die Nahrung besteht wohl grösstenteils aus Plankton, seien es lebendige Organismen oder Detritus von höheren Pflanzen. Die Larven können aber auch andere Nahrung zu sich nehmen, wie diejenigen, welche ich in Tjibodas züchtete, die sich nur von Fadenalgen ernährten und sich dennoch normal entwickelten.

TREUB-Laboratorium.

Buitenzorg, im Mai 1921.

\section{ZITIERTE LITERATUR.}

N. Annandale. The Structure and Mechanism of the Funnel surrounding the Mouth in the Tadpole of Megalophrys montana. Fasciculi Malayenses. Zoology. Part. II, 1903.

- Notes on the Freshwater Fauna of India. VIII. Some Himalayan Tadpoles. Journ. Asiat. Soc. of Bengal. Vol. II, 1906.

G. A. Boulenger. Report on the Batrachians and Reptiles. Fasciculi Malayenses. Zoology. Part. I, 1903.

- A Revision of the Oriental Pelobatid Batrachians (Genus Megalophrys) Proc. Zool. Soc. London, 1908.

- Reptilia and Batrachia in: A Vertebrate Fauna of the Malay Peninsula. 1912

HANS GADOW. Amphibia and Reptiles in: The Cambridge Natural History. 1909.

P. N. VAN KAmPEN. Beitrag zur Kenntnis der Amphibien-larven des Indischen Archipels. Natuurk. Tijdschr. voor Ned. Indië. Deel LXIX, 1909.

MAX Weber. Ueber auffallende Ecaudatenlarven von Tjibodas (Java). Ann. Jard. Bot. Buitenzorg. 2me Suppl. 1898.

FRANZ WERNER. Lurche und Kriechtiere in: Brehms Tierleben. 1912. 